\title{
Perfil nutricional de niñas y niños indígenas menores de cinco años del Paraguay y su asociación con factores socioeconómicos y otros determinantes sociales, Encuesta de Hogares Indígenas 2008
}

\author{
Nutritional profile of indigenous girls and boys under five \\ years of age in Paraguay and its association with \\ socioeconomic factors and other social determinants, \\ Indigenous Household Survey 2008
}

\author{
Vit Bubak' ${ }^{1}$ Marta Sanabria ${ }^{2}$, Susana Sánchez Bernal ${ }^{2}$, Norma Medina $^{3}$
}

\section{RESUMEN}

Introducción: La desnutrición es un desafío pendiente que influye nocivamente en el desarrollo de los niños indígenas, afectando su potencial de crecimiento. Objetivo: Analizar la situación nutricional niños indígenas menores de cinco años en el Paraguay y su asociación con factores socioeconómicos y otros determinantes sociales. Materiales y Métodos: Estudio transversal, descriptivo, analítico, realizado con datos representativos a nivel nacional de la Encuesta de Hogares Indígenas (EHI 2008). Variables: edad, sexo, grupo lingüístico, peso, talla, acceso a agua, acceso a saneamiento básico, tenencia de seguro médico, peso al nacer, lactancia materna, episodios de diarrea, educación. Se consideraron los siguientes grupos de edad: menores de un año, de 1 a 2 años, 2 a 3 años, 3 a 4 años, 4 años y más. El diagnóstico nutricional fue por antropometría, según criterios de la OMS: puntaje z Peso/Edad, Peso/Talla y Talla/Edad. Estadísticas: Se utilizaron promedio, desviación estándar, porcentajes. También se utilizó t Student y Chi cuadrado de Pearson. Significancia $\mathrm{p}<0,05$. Resultados: Fueron estudiados 555 niñas y niños menores de cinco años, edad promedio 29,1 meses (1-59 meses), 53,9\% varones. Los promedios de zPeso/Edad, de zPeso/Talla y de zTalla/Edad fueron 0,56 $\pm 1,2 \mathrm{DE} ; 0,64 \pm 1,2 \mathrm{DE} ;-1,75 \pm 1,6 \mathrm{DE}$, respectivamente. La prevalencia de Desnutrición Global (DG) fue del 9,8\% (en riesgo del 25,0\%), de Desnutrición Aguda (DA) del 1,5\% (en

\section{ABSTRACT}

Introduction: Malnutrition is a pending challenge that negatively influences the development of indigenous children, affecting their potential for growth. Objective: To analyze the nutritional status of indigenous children under five years of age in Paraguay and its association with socio-economic factors and other social determinants. Materials and Methods: This was a crosssectional, descriptive, analytical study, using representative data from the nation-wide Indigenous Household Survey (IHS 2008). Variables were: Age, sex, linguistic group, weight, height, access to water, access to basic sanitation, medical insurance, birth weight, breastfeeding, episodes of diarrhea and education. We used the following age groups in our study: under one year of age, ages 1 to 2 years, 2 to 3 years, 3 to 4 years and over 4 years of age. The nutritional diagnosis was made by anthropometry, according to WHO criteria: z score Weight / Age, Weight / Height and Height / Age. Statistics: Average, standard deviation and percentages were used. Student's t test and Pearson's Chi square were also used. Significance was defined as $\mathrm{p}<0.05$. Results: 555 girls and boys under 5 years of age were studied, the average age 29,1 months (1-59 months), 53,9\% were males. The averages of Weight / Age, of Weight / Height and of Height/ Age were -0,56 $\pm 1,2 \mathrm{SD} ; 0,64 \pm 1,2 \mathrm{DE} ;-1,75 \pm 1,6 \mathrm{DE}$, respectively. The prevalence of Global Malnutrition (GM)

\footnotetext{
${ }^{1}$ Instituto Desarrollo. Asunción, Paraguay

${ }^{2}$ Universidad Nacional de Asunción. Facultad de Ciencias Médicas. Cátedra y Servicio de Pediatría. Asunción, Paraguay.

${ }^{3}$ Dirección General de Estadística, Encuestas y Censos (DGEEC). Asunción, Paraguay.

Correspondencia: Marta Cristina Sanabria. Correo: marta.sanabria@gmail.com

Conflicto de interés: Los autores declaran no poseer conflicto de interés

Recibido: 16/04/2018. Aceptado: 29/04/2018

DOI: https://doi.org/10.31698/ped.45012018004
} 
riesgo del 5,6\%), de Desnutrición Crónica (DC) del 41,7\% (en riesgo del $29,4 \%$ ), y de Obesidad del 9,0\% (en riesgo del $28,6 \%$ ). No se observó asociación significativa entre el estado nutricional y sexo. Se observó asociación significativa entre los grupos de edad y la DA $(p<0,05)$, la DC $(p<0,01)$, y el riesgo de DG $(p<0,05)$. Se observó asociación significativa entre los grupos lingüísticos y la $\mathrm{DC}$, el riesgo de DG, y el sobrepeso $(\mathrm{p}<0,05)$. Hubo mayor desnutrición en niños: sin seguro médico (DC 25,0 vs $43,4 \%$, p<0,01), parto domiciliario (DC 33,2 vs 45,3\%; DG 5,0 vs $11,8 \%$; $<<0,05$ ), con bajo peso de nacimiento (en riesgo de DG 14,5 vs 39,9\%; p<0,05), con diarrea durante los 3 meses anteriores (en riesgo de DG 21,2 vs 34,7\%, p<0,01; sobrepeso 21,0 vs $31,6 \%$, p $<0,01$ ), con madre no-alfabetizada (en riesgo de DG 18,6 vs 30,3\%, p<0,01), con menor acceso a agua segura (DC vs $42,8 \mathrm{vs} 27,6 \% \mathrm{p}<0,01$ y con piso de tierra (en riesgo de DA 0,9 vs 6,2\%, $\mathrm{p}<0,05$; DG 0,4 vs $11,0 \%, \mathrm{p}<0,01)$. Conclusiones: Existe una alta prevalencia de desnutrición crónica en niños indígenas menores de cinco años de edad. Las políticas e intervenciones de alimentación y nutrición diseñadas para los pueblos indígenas deben seguir adaptándose de acuerdo con los estilos de vida culturales y las percepciones alimentarias de las comunidades para mejorar esta situación.

Palabras claves: América Latina, desnutrición, estado nutricional, factores socio-económicos, niños, población indígena.

\section{INTRODUCCIÓN}

La evidencia científica en varios países de América Latina ha demostrado que las disparidades nutricionales varían significativamente entre las poblaciones indígenas y no indígenas ${ }^{(1)}$. Estas disparidades se deben entre otras causas a que los pueblos indígenas se encuentran entre los segmentos más marginados política y socioeconómicamente, con menor acceso a la educación, seguridad alimentaria, salud, y servicios básicos ${ }^{(2,3,4)}$.

La desnutrición constituye de los problemas prioritarios de salud pública. Es responsable de la tasa de mortalidad más alta en los niños, menor potencial de desarrollo, mayor riesgo en la etapa adulta de enfermedades crónicas (obesidad, diabetes, enfermedades cardiovasculares, entre otras) ${ }^{(5)}$. Muchos de estos efectos son consistentes con los hallazgos de análisis comparativos de los niveles de salud que apuntan a mayores tasas de mortalidad y morbilidad entre las poblaciones was $9.8 \%$ (at risk of $25.0 \%$ ), of Acute Malnutrition (AM) was $1.5 \%$ (at risk of $5.6 \%$ ), of Chronic Malnutrition (CM) was $41.7 \%$ (at risk of $29.4 \%$ ), and of Obesity was $9.0 \%$ (at risk of $28.6 \%$ ). No significant association was observed between nutritional status and sex. There was a significant association between age groups and AM $(\mathrm{p}<0.05), \mathrm{CM}$ $(\mathrm{p}<0.01)$, and risk of GM $(\mathrm{p}<0.05)$. There was a significant association between linguistic groups and $\mathrm{CM}$, the risk of GM, and overweight $(p<0.05)$. There was greater malnutrition in children without medical insurance (CM 25.0 vs $43.4 \%$, p < 0.01), who had a home delivery (CM 33.2 vs $45.3 \%$; GM 5.0 vs $11.8 \%$; $<<0.05$ ), with low birth weight (at risk of GM 14.5 vs $39.9 \% ; \mathrm{p}<0.05$ ), with diarrhea during the previous 3 months (at risk of GM 21.2 vs $34.7 \%$, $p<0.01$, overweight 21.0 vs $31.6 \%, p<0.01$ ), with a non-literate mother (at risk of GM 18.6 vs $30.3 \%$, $<<0.01$ ), with less access to safe water (CM vs 42.8 vs $27.6 \% \mathrm{p}<0.01$ ) and with a dirt floor (at risk of AM 0.9 vs. $6.2 \%$, $p<0.05$, DG 0.4 vs. $11.0 \%, \mathrm{p}<0.01)$. Conclusions: There is a high prevalence of chronic malnutrition in indigenous children under the age of 5. Food and nutrition policies and interventions designed for indigenous peoples must continue to adapt in accordance with cultural lifestyles and the food perceptions of communities to improve this situation.

Keywords: Latin America, malnutrition, nutritional status, socio-economic factors, children, indigenous population.

indígenas en comparación con la población no indígena en América Latina ${ }^{(2,3,6)}$. En el caso de Paraguay, las tasas de mortalidad neonatal e infantil para las poblaciones indígenas son mayores que las indicadas para la población nacional ${ }^{(7)}$.

La desnutrición en los primeros años de vida también puede tener consecuencias a largo plazo para el desarrollo de las capacidades humanas, ya que se ha relacionada con un menor desarrollo mental y logros escolares deficientes. Además de las graves consecuencias para la salud de una persona, la economía también se ve afectada por la desnutrición, porque la alta prevalencia de esta condición obstaculiza el desarrollo económico y perpetúa la pobreza tanto directamente, a través de una pérdida de productividad debido a la mala condición física, e indirectamente, a través de una función pobre cognitiva y déficit de aprendizaje ${ }^{(5)}$. 
El objetivo de esta investigación es analizar la situación nutricional niños indígenas menores de cinco años en el Paraguay durante el periodo 2008 y su asociación con factores socio-económicos y otros determinantes sociales.

\section{MATERIAL Y MÉTODOS}

El presente estudio analiza datos de la Encuesta de Hogares Indígenas 2008 (EHI 2008). La encuesta fue implementada entre mayo y junio de 2008 por la Dirección General de Estadística, Encuesta y Censos $(\text { DGEEC })^{(8)}$. La población indígena en Paraguay consta de 18 grupos étnicos organizados en 5 familias lingüísticas: Guaikuru, Guaraní, Maskoy, Matako/Mataguayo, y Zamuco.

La encuesta estuvo dirigida a la población que reside en comunidades indígenas de los 17 departamentos del país. Los hogares se seleccionaron a través de un diseño bietápico y probabilístico. La unidad primaria de muestreo fue una comunidad indígena seleccionada al interior de cada etnia y familia lingüística en forma sistemática, aleatoria y con probabilidad proporcional al tamaño. El listado de viviendas existentes dentro de cada comunidad seleccionada se convirtió en la base para la segunda etapa de muestreo. Un total de 926 viviendas fueron seleccionadas durante la última etapa con la ayuda de los líderes de la comunidad.

Solo los hogares con niños menores de 5 años fueron considerados para el análisis. La muestra final incluyó un total de 555 niños, distribuidos a través de 380 viviendas distintas y 73 comunidades indígenas. Teniendo en cuenta el diseño de muestra, la estimación de población para las diferentes variables que investiga la encuesta se obtiene multiplicando la variable o característica de cada persona por su correspondiente factor de expansión. Los factores de ponderación resultan de las proyecciones de población para cada familia. La mayoría de los niños analizados en este estudio (3 de cada 5) pertenecían a la familia lingüística Guaraní $(44,7 \%)$ o a la familia lingüística Matako Mataguayo $(24,3 \%)$. Todos los 380 hogares considerados en este estudio fueron encontrados en áreas rurales.
Para evaluar el estado nutricional de los niños, se usaron los índices antropométricos de peso para la talla $(\mathrm{P} / \mathrm{T})$, talla para la edad $(\mathrm{T} / \mathrm{E})$, y peso para la edad (P/E), expresados como puntajes de desviación estándar (puntajes $z$ o $z$-scores), según el estándar recomendado por la Organización Mundial de la Salud (OMS). Los niños cuyo $z \mathrm{P} / \mathrm{T}$ estaba por debajo de menos dos desviaciones estándar $(z \mathrm{P} / \mathrm{T}<-2 \mathrm{DE})$ del peso promedio de la población estándar de referencia fueron clasificados como desnutrición aguda, mientras que los niños cuyo $z \mathrm{P} / \mathrm{T}$ estaba dentro del intervalo $(-2 \mathrm{DE} \leq z \mathrm{P} / \mathrm{T}<-1 \mathrm{DE})$ fueron considerados en riesgo de desnutrición aguda. Por el contrario, los niños cuyo $\mathrm{P} / \mathrm{T}$ estaba por encima de dos desviaciones estándar $(z \mathrm{P} / \mathrm{T}>2 \mathrm{DE})$ fueron clasificados como obeso, mientras que los niños cuyo $z \mathrm{P} / \mathrm{T}$ estaba dentro del intervalo $(1 \mathrm{DE} \leq \mathrm{zP} / \mathrm{T}<2$ $\mathrm{DE})$ fueron considerados en riesgo de obesidad. Los niños cuyo $z \mathrm{~T} / \mathrm{E}$ estaba por debajo de menos dos desviaciones estándar $(z \mathrm{~T} / \mathrm{E}<-2 \mathrm{DE})$ fueron clasificados como desnutrición crónica, mientras que los niños cuyo $\mathrm{T} / \mathrm{E}$ estaba dentro del intervalo $(-2 \mathrm{DE} \leq z \mathrm{~T} / \mathrm{E}<-1 \mathrm{DE})$ fueron considerados en riesgo de desnutrición crónica. Por último, los niños cuyo $z \mathrm{P} / \mathrm{E}$ estaba por debajo de menos dos desviaciones estándar $(z \mathrm{P} / \mathrm{E}<-2 \mathrm{DE})$ fueron clasificados como desnutridos (desnutrición global), mientras que los niños cuyo $z \mathrm{P} / \mathrm{E}$ estaba dentro del intervalo $(-2 \mathrm{DE} \leq$ $z \mathrm{P} / \mathrm{E}<-1 \mathrm{DE})$ fueron considerados en riesgo de desnutrición global. Todos los indicadores antropométricos fueron construidos utilizando el software WHOAnthro, versión 3.2.2 (OMS).

Se realizó un análisis descriptivo de los indicadores antropométricos y de la prevalencia de desnutrición (general y estratificado por sexo y grupos de edad) para el conjunto de niños y por los grupos lingüísticos. Se consideraron cinco grupos de edad: menores de 1 año (< 1 año), 1 año a 2 años, 2 añosa3 años, 3 años a 4 años, y 4 años y más. Además del análisis descriptivo, se evaluaron también las asociaciones entre las prevalencias de desnutrición y los factores de nacimiento (lugar del nacimiento del niño, bajo peso al nacer del niño), los indicadores de alimentación neonatal y post-neonatal (lactancia materna exclusiva durante los primeros seis meses de vida del niño, lactancia no exclusiva durante los primeros doce meses de vida del niño, y la introducción de alimentos sólidos antes de los 6 
meses de edad del niño), los indicadores de salud (cobertura por el seguro de salud, episodio reciente de diarrea), escolaridad materna/paterna, y los indicadores de saneamiento básico en el hogar (origen de agua, tipo de saneamiento básico, y presencia del piso de tierra en el hogar).

Las variables continuas se expresaron en términos de promedios \pm desviaciones estándares (DE). Las variables categóricas se expresaron como porcentajes. La significancia de las diferencias entre los valores promedios de la altura, del peso, y del puntaje $z$ se evaluó utilizando el test de $t$, basado en los errores estándar robustos agrupados a nivel de la comunidad; la significancia conjunta fue determinada por la prueba de Wald. Para evaluar asociación entre las variables categóricas, se utilizó la prueba de chicuadrado de Pearson, con la corrección de segundo orden de Rao y Scott ${ }^{(9)}$. Todas las estadísticas se calcularon teniendo en cuenta el diseño de la muestra, utilizando el conjunto de comandos svy de Stata ${ }^{(10)}$. El nivel de significancia se estableció en $p<0,05$; como referencia, se informan los valores $p$ hasta $p=$ 0.10 . Todos los procedimientos estadísticos fueron realizados con el programa estadístico Stata/IC versión $14.2^{(11)}$.

\section{RESULTADOS}

Fueron estudiados 555 niños y niñas, de los cuales $53.9 \%(n=299)$ fueron varones (Tabla 1$)$. La edad promedio fue de 29,1 meses (2 años y 5,1 meses); la mediana de edad fue de 28.5 meses (el $5^{\circ}$ percentil de 3,1 meses y el $95^{\circ}$ percentil de 56,0 meses). La altura promedio fue de $82,2 \mathrm{~cm}$ y el peso promedio fue de $11,7 \mathrm{~kg}$. Las diferencias por sexo no fueron estadísticamente significativas $(p=0,18$ y $p=0,08)$. Se observa en la Tabla 1 otras características antropométricas de los niños indígenas según sexo y edad (menores de dos años y iguales o mayores de dos años).

Tabla 1. Características antropométricas de los niños indígenas según sexo y edad. EIH Indígena 2008. Valores promedios y desviación estándar.

\begin{tabular}{lccccc}
\hline & Todos & Niños & Niñas & $<$ 2 Años & $\geq$ 2 Años \\
\hline Edad (meses) & $29,1(16,8)$ & $29,3(16,7)$ & $28,9(17,0)$ & $12,1(6,6)$ & $41,1(10,2)$ \\
Altura (cm) & $82,2(13,4)$ & $83,1(13,7)$ & $81,2(13,1)$ & $69,7(8,3)$ & $91,1(8,4)$ \\
Peso (Kg) & $11,7(3,6)$ & $12,0(3,6)$ & $11,4(3,5)^{\mathrm{a}}$ & $8,5(2,1)$ & $14,1(2,4)$ \\
$\mathbf{N}^{\circ}$ de niños & 555 & 299 & 256 & 222 & 333 \\
\hline
\end{tabular}

${ }^{a}$ Nivel de significancia de la diferencia entre sexos: $p=0,080$
En la Tabla 2. se presentan los promedios de los puntajes zPeso/Edad (zP/E), Peso/Talla (zP/T), y Talla/Edad (zT/E) para toda la muestra, así como según sexo y grupo de edad. Se pudo observar que para ninguno de los tres puntajes $\mathrm{z}$, la diferencia entre niños y niñas fue estadísticamente significativa.

Tabla 2. Promedios del puntaje $\mathrm{z}$ según sexo y grupos de edad en niños indígenas menores de cinco años de edad. (Pro medio y desviación estándar)

\begin{tabular}{lccccc}
\hline & Todos & Niños & Niñas & $<2$ Años & $\geq 2$ Años \\
\hline zPeso/Edad (z P/E) & $-0,56(1,2)$ & $-0,56(1,2)$ & $-0,57(1,1)$ & $-0,54(1,3)$ & $-0,58(1,0)$ \\
zPeso/Talla (zP/T) & $0,64(1,2)$ & $0,63(1,1)$ & $0,64(1,3)$ & $0,45(1,5)$ & $0,77(1,0)^{*}$ \\
zTalla/Edad (zT/E) & $-1,75(1,6)$ & $-1,74(1,5)$ & $-1,74(1,5)$ & $-1,53(1,7)$ & $-1,90(1,5)^{*}$ \\
\hline * Diferencia significativa entre las edades: $\mathbf{p}<0,05$.
\end{tabular}

En la Tabla 3 se presentan las prevalencias de la desnutrición. Si bien la prevalencia de desnutrición aguda fue relativamente baja $(1,5 \%)$, un número mayor de niños presentaban riesgo de desnutrición aguda (5,6\%). Se observó un patrón similar de resultados para niñas y niños, aunque no se encontraron diferencias significativas en la prevalencia de desnutrición aguda o el riesgo de la misma entre los dos grupos. Finalmente, la prevalencia de desnutrición aguda fue significativamente mayor entre los niños menores de 2 años que entre los niños mayores de 2 años ( $3,6 \%$ vs. $0.0 \%, \mathrm{p}<0,05)$.

Tabla 3. Prevalencia de desnutrición (DNT) y obesidad según sexo y grupos de edad en niños indígenas menores de cinco años de edad. EIH Indígena 2008.

\begin{tabular}{|c|c|c|c|c|c|c|}
\hline & Valor de Referencia & Todos & Niños & Niñas & $<2$ Años $>$ & $\geq 2$ Años \\
\hline DNT Aguda & $\mathrm{zP} / \mathrm{T}<-2 \mathrm{DE}$ & 1,5 & 1,0 & 2,0 & 3,6 & $0,0 * *$ \\
\hline En riesgo & $-2 \mathrm{DE} \leq \mathrm{zP} / \mathrm{T}<-1 \mathrm{DE}$ & 5,6 & 5,9 & 5,2 & 8,5 & $3,5^{\mathrm{a}}$ \\
\hline DNT Crónica & $z T / E<-2 D E$ & 41,7 & 42,0 & 41,4 & 38,1 & 44,3 \\
\hline En riesgo & $-2 \mathrm{DE} \leq \mathrm{zT} / \mathrm{E}<-1 \mathrm{DE}$ & 29,4 & 31,0 & 27,6 & 30,6 & 28,5 \\
\hline DNT Global & $z P / E<-2 D E$ & 9,8 & 10,1 & 9,5 & 12,0 & 8,2 \\
\hline En riesgo & $-2 \mathrm{DE} \leq \mathrm{zP} / \mathrm{E}<-1 \mathrm{DE}$ & 25,0 & 24,0 & 26,1 & 26,2 & 24,1 \\
\hline Obesidad & $\mathrm{z} P / \mathrm{T}<2 \mathrm{DE}$ & 9,0 & 8,9 & 9,0 & 11,7 & $7,0^{*}$ \\
\hline Sobrepeso & $-1 \mathrm{DE} \leq \mathrm{zP} / \mathrm{T}<2 \mathrm{DE}$ & 28,6 & 27,1 & 30,3 & 25,7 & 30,6 \\
\hline
\end{tabular}

En cuanto a la prevalencia de desnutrición crónica, se observó que más que cuatro niños de diez $(41,7 \%)$ estaban crónicamente desnutridos. Además, tres niños de diez $(29,4 \%)$ estaban en riesgo de la 
desnutrición crónica. No se observó una diferencia significativa en la prevalencia de desnutrición crónica ni entre niñas y niños, ni entre los grupos de edad.

La prevalencia de desnutrición global alcanzó el 9,8\%; sin embargo, como en el caso de la desnutrición aguda, un número significativamente mayor de niños estaba en riesgo de la desnutrición global (25\%). No se encontraron diferencias significativas ni en la prevalencia de desnutrición global ni en su riesgo entre niñas y niños, o entre grupos de edad.

Los resultados para la obesidad son tanto cuantitativamente como cualitativamente similares a los de la desnutrición global, aunque se puede observar que hubo un número ligeramente mayor de niños menores de dos años que fueron obesos en comparación con los niños mayores de dos años $(11,7 \%$ vs. $7,0 \%$, $p$ $=0,057)$.

La Tabla 4 muestra las prevalencias de la desnutrición según grupos de edad. Se consideraron cinco grupos de edad: menores de 1 año ( $<1$ año), 1 año a 2 años, 2 años a 3 años, 3 años a 4 años, y 4 años y más. Se observó una asociación significativa entre los grupos de edad y la desnutrición aguda $(\mathrm{p}<0,05)$, la desnutrición crónica $(\mathrm{p}<0,01)$, y el riesgo de desnutrición global $(\mathrm{p}<0,05)$. Los niños de 1 año a 2 años tuvieronademás de la mayor prevalencia de desnutrición aguda $(3,6 \%)$ - también la mayor prevalencia de desnutrición crónica $(55,1 \%)$ de todos los grupos de edad, aunque a niveles estadísticamente no significativos en la mayoría de los casos. Además, el mismo grupo también exhibió el mayor riesgo de la desnutrición aguda $(11,7 \%)$ y desnutrición global $(33,8 \%)$, aunque en niveles marginales de significancia $(\mathrm{p}=$ $0,08$ y 0,07$)$. Los niños menores de 1 año tuvieron a la vez la menor prevalencia de desnutrición crónica $(20,3 \%)$ en relación con otros grupos de edad, pero también la mayor prevalencia de obesidad $(15,8 \%)$.

En la Tabla 5 se presentan los promedios de los puntajes z Peso/Edad (z P/E), Peso/Talla (z P/T), y Talla/Edad (z T/E) por grupos lingüísticos. Los resultados muestran una relación estadísticamente significativa entre cada uno de los tres promedios de los puntajes z y los grupos lingüísticos. El grupo lingüístico Guaraní- el más grande de los cinco grupos - exhibe los puntajes z P/E y z T/E más bajos entre los grupos lingüísticos. En particular, su z P/E $(-0,71)$ es significativamente más pequeño que el z $\mathrm{P} / \mathrm{E}$ de cualquier otro grupo lingüístico $(\mathrm{p}<-0,05)$ con la excepción de Zamuco $(\mathrm{p}<-0,09)$; y su z T/E $(-1,88)$ es significativamente más pequeño que los z T/E de Matako Mataguayo y Guaikuru $(p<0,05)$, pero solo marginalmente más pequeño que el z T/E de Maskoy $(\mathrm{p}=0,052)$ y Zamuco (diferencia no significativa). El grupo lingüístico Matako Mataguayo tiene el zP/T más alto, aunque su puntaje no es significativamente diferente no es diferente de otros grupos con la excepción del grupo Guaraní $(\mathrm{p}<0,05)$.

Tabla 4. Prevalencia de por déficit y por exceso, según grupos de edad, en niños indígenas menores de cinco años de edad. EIH Indígena 2009.

\begin{tabular}{|c|c|c|c|c|c|c|c|}
\hline & Valor de Referencia & $<1$ Año & $\begin{array}{l}\text { 1Año a } \\
\text { 2Años }\end{array}$ & $\begin{array}{c}\text { 2Años a } \\
\text { 3Años }\end{array}$ & $\begin{array}{c}\text { 3Años a } \\
\text { 4Años }\end{array}$ & $\begin{array}{c}\geq 4 \\
\text { Años }\end{array}$ & $\begin{array}{c}\text { X2 } \\
\text { p-val }\end{array}$ \\
\hline DNT Aguda & $\mathrm{zP} / \mathrm{T}<-2 \mathrm{DE}$ & $3,6^{a}$ & $3,6^{\#}$ & 0,0 & 0,0 & 0,1 & $*$ \\
\hline En riesgo & $-2 \mathrm{DE} \leq \mathrm{zP} / \mathrm{T}<-1 \mathrm{DE}$ & 5,7 & $11,2^{\mathrm{b}}$ & 1,7 & 5,2 & 3,8 & \\
\hline DNT Crónica & $z T / E<-2 D E$ & $20,3^{\#}$ & $55,1^{\mathrm{c}}$ & 47,4 & 45,4 & 39,0 & $* *$ \\
\hline En riesgo & $-2 \mathrm{DE} \leq \mathrm{zT} / \mathrm{E}<-1 \mathrm{DE}$ & 33,8 & 27,6 & 27,6 & 26,6 & 31,9 & \\
\hline DNT Global & $z P / E<-2 D E$ & 10,1 & $13,8^{\mathrm{d}}$ & 9,3 & 4,2 & 11,5 & \\
\hline En riesgo & $-2 \mathrm{DE} \leq \mathrm{zP} / \mathrm{E}<-1 \mathrm{DE}$ & $18,4^{\mathrm{e}}$ & $33,8^{f}$ & 23,2 & 23,1 & 26,5 & $*$ \\
\hline Obesidad & $\mathrm{zP} / \mathrm{T}<2 \mathrm{DE}$ & $15,8^{*}$ & 7,9 & 7,1 & 11,6 & 1,5 & \\
\hline Sobrepeso & $1 \mathrm{DE} \leq \mathrm{zP} / \mathrm{T}<2 \mathrm{DE}$ & 30,6 & 21,0 & $25,9^{\mathrm{h}}$ & 37,5 & 28,7 & \\
\hline$N^{\circ}$ de niños & & 105 & 117 & 118 & 116 & 99 & \\
\hline \multicolumn{8}{|c|}{ 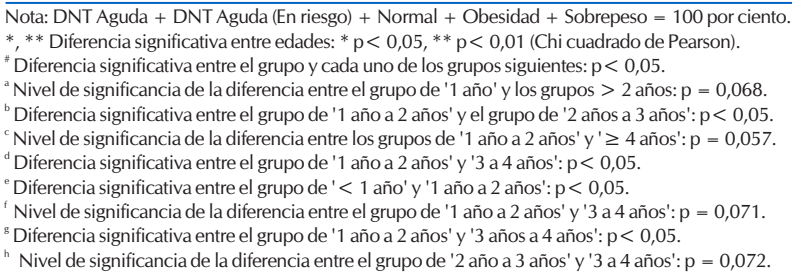 } \\
\hline
\end{tabular}

Tabla 5. Promedios del puntaje z y desviación estándar por grupos lingüísticos en niños indígenas menores de cinco años de edad. EIH Indígena 2008

\begin{tabular}{|c|c|c|c|c|c|c|}
\hline & Guaraní & $\begin{array}{c}\text { Matako } \\
\text { Mataguayo }\end{array}$ & Maskoy & Zamuco & Guaikuru & p-val \\
\hline zPeso/Edad (z P/E) & $-0,71(1,1)^{a}$ & $-0,14(1,1)$ & $-0,32(1,2)^{b}$ & $-0,32(1,5)$ & $-0,05(0,8)$ & $* *$ \\
\hline zPeso/Talla (zP/T) & $0,53(1,2)^{c}$ & $0,98(1,1)$ & $0,78(1,4)$ & $0,91(1,5)$ & $0,76(1,0)$ & $* *$ \\
\hline zTalla/Edad (zT/E) & $-1,88(1,6)^{d}$ & $-1,45(1,6)$ & $-1,48(1,5)$ & $-1,62(1,6)^{\mathrm{e}}$ & $-1,09(1,0)$ & $*$ \\
\hline $\mathrm{N}^{\circ}$ de niños & 248 & 135 & 93 & 59 & 20 & 555 \\
\hline \multicolumn{7}{|c|}{ 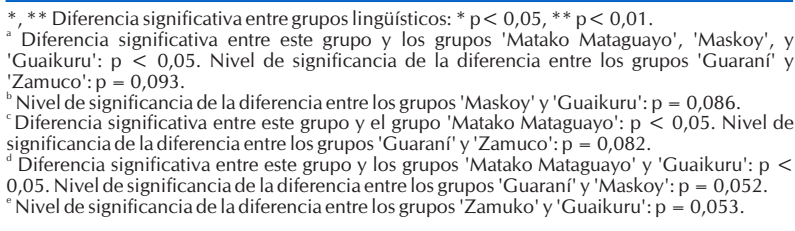 } \\
\hline
\end{tabular}


En la Tabla 6 se presentan los resultados del análisis de la prevalencia de desnutrición por grupos lingüísticos. En cuanto a la prevalencia de desnutrición aguda, no se observó una relación estadísticamente significativa entre esta y los grupos lingüísticos. Aunque el grupo lingüístico Zamuco exhibe la prevalencia de desnutrición la más alta $(5,1 \%)$, las diferencias en prevalencias de desnutrición entre los grupos lingüísticos son, en sus mayorías, no significativas. En cuanto a la prevalencia de riesgo de la desnutrición aguda, ésta es más alta en el grupo lingüístico Guaraní (6,9\%).

Niños de los grupos lingüísticos Guaraní y Zamuco registran las prevalencias más altas de la desnutrición crónica $(46,4 \%$ y $39,0 \%)$ y de la desnutrición global (11,7\% y 10,2\%) entre los cinco grupos lingüísticos. Se destaca que el grupo lingüístico Guaraní también exhibe la prevalencia de riesgo de la desnutrición global más alta de los grupos lingüísticos $(29,5 \%)$, casi dos veces más alta que cualquier otro grupo lingüístico. En otras palabras, más de 4 de cada 10 niños guaraní están globalmente desnutridos o en riesgo de desnutrición.

Al mismo tiempo, niños de los grupos lingüísticos Guaraní y Guaikuru registran la prevalencia más baja de la obesidad entre los grupos lingüísticos, aunque en este caso, la diferencia solo es estadísticamente significativa entre los grupos lingüísticos Guaraní y Maskoy $(\mathrm{p}<0,05)$ y marginalmente entre los grupos lingüísticos Guaraní y Zamuco $(\mathrm{p}=0,054)$. La prevalencia de sobrepeso es muy parecida entre los grupos lingüísticos.

La Figura 1 Proporciona datos complementarios de las prevalencias de desnutrición aguda y de obesidad/sobrepeso, definidos por valores límites de los puntajes Peso/Talla $(z \mathrm{P} / \mathrm{T})$, que además muestra el porcentaje de la población de niños con valores de $z \mathrm{P} /$ T normales. Se observa en la figura que los grupos lingüísticos están ordenados por el porcentaje de niños con valores de $z \mathrm{P} / \mathrm{T}$ normales. Es destacar que, en su totalidad (y considerando que las prevalencias de sobrepeso entre los grupos lingüísticos no son estadísticamente significativamente diferentes), la desnutrición afecta alrededor de la mitad de niños de cada grupo lingüístico, siendo las más afectadas las poblaciones de Maskoy, Matako Mataguayo y
Zamuco.

Tabla 6. Prevalencia de malnutrición por déficit y por exceso según grupos lingüísticos en niños indígenas menores de cinco años de edad. EIH Indígena 2008

\begin{tabular}{|c|c|c|c|c|c|c|c|}
\hline & Valor de Referencia & Guaraní & $\begin{array}{c}\text { Matako } \\
\text { Matag. }\end{array}$ & Maskoy & Zamucc & Juaikuru & $\begin{array}{c}X 2 \\
\text { p-val }\end{array}$ \\
\hline DNT Aguda & $\mathrm{zP} / \mathrm{T}<-2 \mathrm{DE}$ & $1,2^{a}$ & $0,7^{\mathrm{b}}$ & $3,2^{c}$ & $5,1^{\#}$ & 0,0 & \\
\hline En riesgo & $-2 \mathrm{DE} \leq \mathrm{zP} / \mathrm{T}<-1 \mathrm{DE}$ & $6,9^{d}$ & 3,0 & 2,2 & 1,7 & 5,0 & 0,10 \\
\hline DNT Crónica & a $\quad z T / E<-2 D E$ & $46,4^{\mathrm{e}}$ & $37,0^{f}$ & 25,8 & $39,0^{\#}$ & 15,0 & $*$ \\
\hline En riesgo & $-2 \mathrm{DE} \leq \mathrm{zT} / \mathrm{E}<-1 \mathrm{DE}$ & $28,6^{\mathrm{g}}$ & $24,4^{\mathrm{h}}$ & 36,6 & 30,5 & 45,0 & 0,07 \\
\hline DNT Global & $z P / E<-2 D E$ & $11,7^{i}$ & $3,7^{i}$ & $7,5^{\mathrm{k}}$ & $10,2^{\sharp}$ & 0,0 & \\
\hline En riesgo & $-2 \mathrm{DE} \leq \mathrm{zP} / \mathrm{E}<-1 \mathrm{DE}$ & $29,4^{\#}$ & 15,6 & 15,1 & 13,6 & 15,0 & $*$ \\
\hline Obesidad & $\mathrm{zP} / \mathrm{T}<2 \mathrm{DE}$ & $6,5^{1}$ & $14,1^{\mathrm{m}}$ & $16,1^{n}$ & $15,3^{\#}$ & 5,0 & \\
\hline Sobrepeso & $1 \mathrm{DE} \leq \mathrm{zP} / \mathrm{T}<2 \mathrm{DE}$ & 27,8 & 33,3 & 25,8 & 32,2 & 35,0 & $*$ \\
\hline$N^{\circ}$ de niños & & 105 & 118 & 117 & 116 & 99 & \\
\hline
\end{tabular}

Nota: Desnutrición (DNT) Aguda + DNT Aguda (En riesgo) + Normal + Obesidad + Sobrepeso $=100$ por ciento

* Diferencia significativa entre grupos lingüísticos: $p<0,01$ (Chi cuadrado de Pearson).

Diferencia significativa entre este grupo y cada uno de los grupos siguientes: $p<0,05$.

Nivel de significancia de la diferencia entre los grupos 'Guaraní' y 'Guaikuru': $p=0,064$.

Nivel de significancia de la diferencia entre los grupos 'Matako Mataguayo' y 'Zamuko': $p$

'Diferencia significativa entre los grupos 'Maskoy' y 'Guaikuru': $p<0,05$

Nivel de significancia de la diferencia entre los grupos 'Guaraní' y 'Matako Mataguayo': p = ,080. Diferencia significativa entre el grupo 'Guaraní' y los grupos 'Maskoy' y 'Zamuko': $\mathrm{p}<$

Nivel de significancia de la diferencia entre los grupos 'Guaraní' y 'Matako Mataguayo': $p=$ 0,060 . Diferencia significativa entre el grupo 'Guaraní' y los grupos 'Maskoy' y 'Guaikuru': $p<$ 0,05

Nivel de significancia de la diferencia entre los grupos 'Matako Mataguayo' y 'Maskoy': $\mathrm{p}=$ 0,071 . Diferencia significativa entre los grupos 'Matako Mataguayao' y 'Guaikuru': $p<0,05$. ${ }^{8}$ Nivel de significancia de la diferencia entre los grupos 'Guarani' $y$ 'Maskoy': $p=0,080$. Nivel de significancia de la diferencia entre los grupos 'Guarani' $y$ 'Maskoy': $p=0,080$.
Diferencia significativa entre los grupos 'Matako Mataguayo' y 'Maskoy': $p<0,05$. Nivel de significancia de la diferencia entre los grupos 'Matako Mataguayo' $y$ ' 'Guaikuru': $p=0,085$. Diferencia significativa entre el grupo 'Guaraní' y los grupos 'Matako Mataguayo' y 'Guaikuru'. $<0,05$.

Diferencia significativa entre los grupos 'Matako Mataguayo' y 'Guaikuru': $p<0,05$ Diferencia significativa entre los grupos 'Maskoy' $y$ 'Guaikuru': $p<0,05$. 'Diferencia significativa entre los grupos 'Guaraní' $y$ 'Maskoy': $p<0,05$. Nivel de significancia de la diferencia entre los grupos 'Guaraní' $y$ 'Zamuko': $p=0,054$

Nivel de significancia de la diferencia entre los grupos 'Matako Mataguayo' y 'Guaikuru': $\mathrm{p}=$ 0,078

Diferencia significativa entre los grupos 'Maskoy' y 'Guaikuru': $p<0,05$

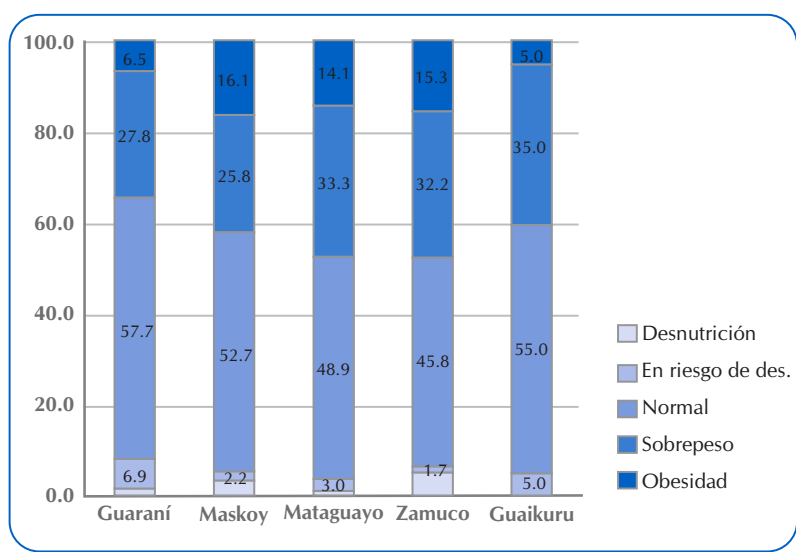

Figura 1. Prevalencia de desnutrición (DNT) por grupos lingüísticos, en niños indígenas menores de cinco años de edad, EIH Indígena 2008 


\section{Factores de nacimiento y seguro médico}

Se observó que sólo el 29,5\% de los niños indígenas nació en el hospital (clínica o sanatorio privado, centro de salud o puesto de salud), y el 70,5\% restante tuvo parto domiciliario. En relación al peso a nacer, el 15,3\% de niños nació con el bajo peso, definido como peso al nacer $<2.500 \mathrm{~g}$. En cuanto al seguro médico, sólo el 9,1\% de los niños indígenas tienen seguro de salud (IPS, seguro privado, seguro local, o sanidad policial).

En la Tabla 7 (Parte A) se presentan los resultados del análisis de la prevalencia de desnutrición por los factores de nacimiento y seguro médico. En cuanto al parto hospitalario, se comprobaron diferencias significativas en las prevalencias de la desnutrición crónica $(33,2 \%$ vs $45,3 \%, \chi 2 \mathrm{p}<0,05)$ y desnutrición global $(5,0 \%$ vs $11,8 \%, \chi 2 \mathrm{p}<0,05)$.En cuanto al bajo peso al nacer, se observó que el bajo peso al nacer fue asociado de la manera significativa con la mayor prevalencia de riesgo de la desnutrición global $(39,9 \%$ vs $14,6 \%, \chi 2 p<0,05)$, y de la manera marginalmente significativa con la mayor prevalencia de la desnutrición aguda $(6,2 \%$ vs $1,1 \%, \chi 2 p<0,07)$. Finalmente, en cuanto al seguro médico, se observó una diferencia significativa en la prevalencia de la desnutrición crónica $(25,0 \%$ vs $43,4 \%, \chi 2 p<0,01)$.

\section{Prácticas alimentarias}

La lactancia materna y la adecuada introducción de la alimentación complementaria son fundamentales para la salud y nutrición óptima del niño ${ }^{(12,13)}$. Por lo tanto, en este estudio también se examinó la asociación entre la desnutrición y la lactancia materna exclusiva durante los primeros seis meses de vida del niño, la lactancia no exclusiva durante los primeros 12 meses de vida del niño, y la introducción de alimentos sólidos antes de los 6 meses de edad. Por último, dado que la contaminación microbiana de los alimentos complementarios es la principal causa de enfermedad diarreica, que es particularmente común en niños de 6 a 12 meses de edad, también se examinó la asociación entre la desnutrición y si el niño ha sufrido recientemente una diarrea ${ }^{(14)}$.

La media de duración de la lactancia materna fue $14,9( \pm 8,9)$ meses (la mediana de 12 meses). Se observó que de la totalidad de los niños, un 2,2\% $(\mathrm{n}=12)$ no había sido nunca amamantado, un $15 \%$ $(\mathrm{n}=83)$ había amamantado menos de 6 meses, un $56,5 \%(n=313)$ entre 4 y 23 meses, y un 26,4\% ( $n=146)$ durante 24 meses o más tiempo. La máxima duración de la lactancia maternal fue 48 meses ( $n=7$ niños).

La edad promedio de incorporación a la dieta de fórmula artificial u otro tipo de leche diferente a la materna fue de 8,3 meses (mínimo 0 mes, máximo 48 meses), excluyendo a aquellos niños que nunca (o todavía) recibieron la leche $(70,1 \%, n=391)$. Mientras que la alimentación complementaria fue iniciada en promedio a los 6,8 meses de edad, excluyendo los casos en los que nunca (o todavía) se inició la alimentación complementaria $(10,3 \%, n=57)$. En este caso, 34\% de los niños/as recibieron alimentación complementaria antes de los 6 meses, y 97\% antes de un año.

Tabla 7. Prevalencia de desnutrición (DNT) vs. indicadores socio-económicos y otros determinantes sociales en niños indígenas menores de cinco años de edad. EIH Indígena 2008

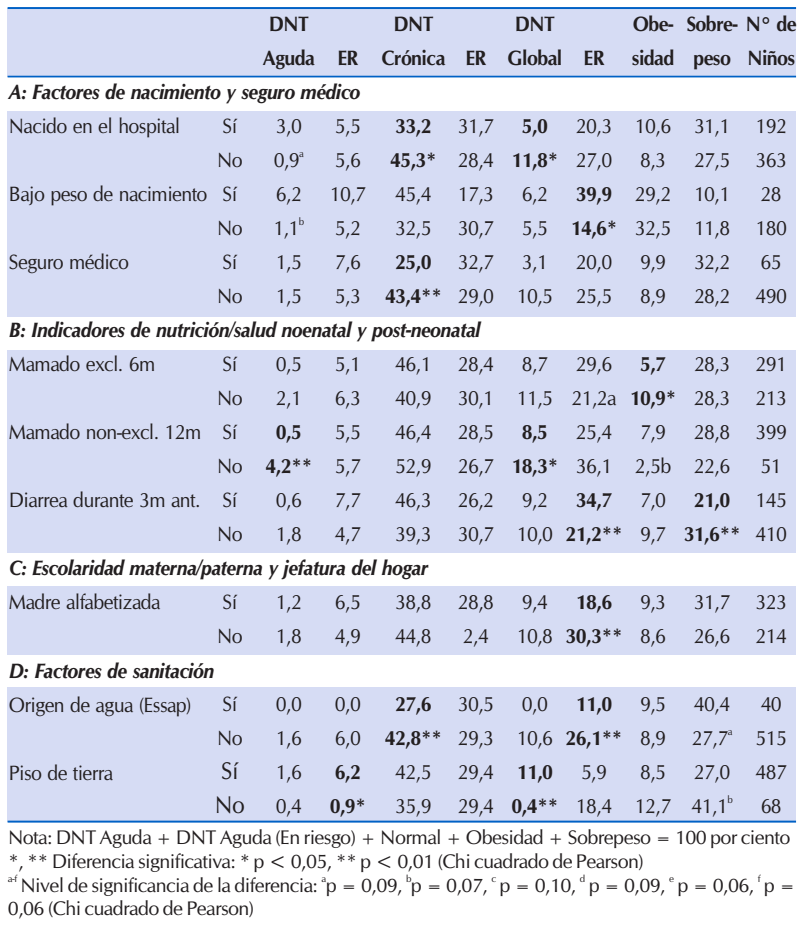

La tasa de lactancia materna exclusiva (definida como lactancia materna sin definida como la práctica de alimentar al lactante únicamente con 
leche materna durante los primeros 6 meses de vida (sin darle ningún otro alimento ni otro tipo de leche durante los seis primeros meses de vida fue $61,2 \%$.

En la Tabla 7 (parte B) se presentan los resultados del análisis de la prevalencia de desnutrición por indicadores de alimentación. En cuanto a la lactancia materna exclusiva, se observó que no hubo diferencias significativas de porcentajes de desnutrición aguda, crónica y global entre niños amamantados y no-amamantados exclusivamente antes de los 6 meses de edad. Sin embargo, se observó diferencia significativa en caso de la obesidad, con los niños del segundo grupo exhibiendo casi el doble de la prevalencia de obesidad que los niños del primer grupo $(10,9 \%$ vs $5,7 \%, \chi 2 \mathrm{p}<0,05)$.

En cuanto a la lactancia materna por los primeros 12 meses, se hallaron diferencias significativas en la prevalencia de desnutrición aguda $(0,5 \%$ vs $54,2 \%, \chi 2 \mathrm{p}<0,01)$, y la desnutrición global $(8,5 \%$ vs $18,3 \%, \chi 2 \mathrm{p}<0,05)$. En cuanto a la incorporación a la dieta de fórmula artificial u otro tipo de leche y la introducción de la alimentación complementaria (antes y después 6 meses) no se comprobaron ningunas diferencias significativas; estos resultados no son reportados, pero son disponibles bajo petición. Finalmente, el reciente episodio de diarrea está asociado con la prevalencia más alta del riesgo de la desnutrición global $(34,7 \%$ vs $21,2 \%, \chi 2$ p $<0,01)$, y con la prevalencia más baja del sobrepeso $(21,0 \%$ vs $31,6 \%, \chi 2 p<0,01)$.

\section{Escolaridad materna/paterna}

Se ha demostrado que la educación de la madre es un determinante de la desnutrición ${ }^{(15,16)}$. El análisis preliminar mostró que el $75,1 \%$ de las madres tuvieron menos de 4 años de educación, de las cuales el 63\% no tuvieron ninguna educación formal; las cifras correspondientes para los padres fueron $63,6 \%$ y $51,0 \%$ respectivamente. Sin embargo, muchas madres y padres con 1 a 3 años de educación no sabían leer ni escribir; del mismo modo, muchos de los que sabían leer y escribir no tuvieron ninguna educación formal. Por último, se analizó también la asociación entre el estado nutricional del niño y si el jefe de hogar fue mujer.
En la Tabla 7 (parte C) se presentan los resultados del análisis de la prevalencia de desnutrición por escolaridad materna. Se observó diferencia significativa solamente en el caso de la prevalencia de riesgo de la desnutrición global; en este caso, los niños con las madres alfabetizadas tenían el riesgo mucho más bajo que los niños con las madres noalfabetizadas (18,6\% vs $30,3 \%)$.

En cuanto a la escolaridad paterna y el sexo del jefe del hogar no se comprobaron ningunas diferencias estadísticamente significativas; estos resultados no son reportados, pero son disponibles bajo petición. Vale la pena señalar que casi todos los demás niños/as $(47,4 \%)$ con bajo peso nació de madres no alfabetizadas (36,9\% de madres sin educación).

\section{Indicadores de saneamiento básico en el hogar}

Respecto al origen de agua para consumo, solo el $7,2 \%$ de los hogares contaba con abastecimiento de agua proveniente de ESSAP (Empresa de Servicios Sanitarios del Paraguay S.A.) o SENASA (Servicio Nacional de Saneamiento Ambiental) $(1,8 \%)$ o de la red comunitaria $(5,4 \%)$, y el 33,3\% se proveía desde pozos (artesianos o con/sin bomba).En el análisis, se utiliza la variable "origen de agua", definida por la presencia o no presencia del agua proveniente de ESSAP/SENASA o de la red comunitaria en el hogar. En cuanto al saneamiento básico, el 89,5\% de los hogares contaba con letrina común o letrina con tapa losa; en tanto que, sólo el 2,7\% de los hogares disponía de baño moderno con pozo ciego. El 8,5\% de los hogares no disponían de ninguno de los tres tipos. En el análisis, se utiliza la variable "saneamiento", definida por la presencia o no presencia de la letrina o baño moderno con pozo ciego en el hogar. Respecto al tipo de piso, $88,5 \%$ de los hogares contaban con el piso de tierra.

En la Tabla 7 (parte D) se presentan los resultados del análisis de la prevalencia de desnutrición por factores de sanitación. En cuanto al origen de agua, se hallaron diferencias significativas en las prevalencias de desnutrición crónica $(27,6 \%$ vs $42,8 \%, \chi 2 \mathrm{p}<0,01)$, y de riesgo de la desnutrición global $(11,0 \%$ vs $26,1 \%, \chi 2 p<0,01)$. En cuanto al tipo de saneamiento, no se comprobaron ningunas diferencias significativas. Finalmente, se observó 
que la presencia del piso de tierra fue asociada de la manera significativa con la mayor prevalencia de riesgo de la desnutrición aguda $(6,2 \%$ vs $0,2 \%, \chi 2$ $\mathrm{p}<0,05)$, y la mayor prevalencia de la desnutrición global $(11,0 \%$ vs $0,4 \%, \chi 2 p<0,01)$.

\section{DISCUSIÓN}

La evidencia científica ha demostrado que - en muchos países de América Latina - las disparidades nutricionales varían significativamente entre las poblaciones indígenas y no indígenas de América Latina. Esta disparidad es muy visible en el caso de la desnutrición crónica, cuya prevalencia tiende a ser aproximadamente el doble en niños indígenas que en niños no indígenas. Por ejemplo, los estudios realizados en Guatemala, demuestran que casi ocho de cada 10 niños indígenas menores de 5 años tenían desnutrición crónica, a diferencia de cuatro de cada 10 niños no indígenas (ladinos); en Ecuador y Perú, más de la mitad de los niños indígenas menores de 5 años tenían desnutrición crónica, en comparación con una cuarta parte de los niños no indígenas; en Bolivia, la prevalencia de la desnutrición crónica en los niños indígenas alcanzó un promedio de $35-40 \%$ en comparación con alrededor del $24 \%$ en los niños no indígenas ${ }^{(1)}$.

El presente estudio revela un escenario nutricional muy desfavorable para los niños indígenas en Paraguay. La prevalencia de desnutrición crónica en niños indígenas $(41,7 \%)$ fue entre tres y cuatro veces mayor que la prevalencia de desnutrición crónica observada en niños no indígenas (17,5\% según los datos de la Encuesta Permanente de Hogares 2005, o del 10,5\% si se basa en la Encuesta de Ingresos y Gastos y Condiciones de Vida/EIG 2011/12) ${ }^{(17,18)}$. Del mismo modo, la prevalencia de desnutrición global en niños indígenas $(9,8 \%)$ resultó ser más de tres veces superior a la prevalencia de desnutrición global observada en niños no indígenas $(3,4 \%$ si se basa en la EPH $2005^{(17)}$, o 2,6\% si se basa en la EIG 2011/12 ${ }^{(18))}$. En ambos casos, las tasas para niños no indígenas consideraron los criterios de evaluación nutricional acorde a las nuevas curvas de crecimiento OMS 2006.

Los datos también mostraron una asociación significativa entre la desnutrición aguda, la desnutrición crónica y el riesgo de desnutrición global y los grupos de edad. La desnutrición aguda estuvo presente $(3,6 \%)$ en los niños menores de 2 años, mientras que casi no estuvo presente en ningún grupo de niños mayores de 2 años. Por otro lado, los niños menores de 1 año tuvieron a la vez la menor prevalencia de desnutrición crónica $(20,3 \%)$ y la mayor prevalencia de obesidad $(15,8 \%)$ en relación con otros grupos de edad. Al relacionar la malnutrición con el género, en este estudio no se encontró asociación. Al observar los cinco grupos lingüísticos distintos de niños indígenas, se observó tasas de prevalencia relativamente más altas de desnutrición crónica y desnutrición global en los grupos lingüísticos de Guaraní y Zamuco.

Es probable que una combinación de factores sea responsable de una mayor prevalencia de desnutrición entre los niños indígenas en Paraguay. En este estudio, se analizó una serie de factores socioeconómicos que ha demostrado estar relacionada con la nutrición infantil, incluida las características ambientales de los hogares, las prácticas de alimentación infantil, y las condiciones socioeconómicas.

Los hallazgos de la EHI 2008 destacan las principales deficiencias en la disponibilidad de servicios públicos para las aldeas indígenas en Paraguay, especialmente cuando se trata de saneamiento básico, agua potable y manejo de desechos sólidos. Como ya se señaló, con respecto a la gestión de los desechos humanos, la infraestructura más típica observada en un hogar indígena era una letrina común, con aguas residuales que rara vez se recolectaban o recibían algún tipo de tratamiento; sólo el 2,7\% de los hogares disponía de baño moderno con pozo ciego. De manera similar, en cuanto al origen de agua para consumo, más del $90 \%$ de los hogares obtenían agua de pozos (33\%) o aljibes/ríos $(60 \%)$; sólo el 7,2\% de los hogares contaba con el abastecimiento de agua proveniente de ESSAP o SENASA (redes nacionales) o de la red comunitaria.

Los resultados mostraron que la prevalencia de desnutrición crónica y el riesgo de desnutrición global fue significativamente mayor en hogares sin abastecimiento de agua proveniente de ESSAP o SENASA o de la red comunitaria $(60 \%$ mayor prevalencia en caso de desnutrición crónica y 140\% 
mayor prevalencia en caso de riesgo de desnutrición global); sin embargo, el saneamiento en sí mismo no tuvo ningún efecto sobre la malnutrición.

Además de estas deficiencias, también se demostró que la presencia de piso de tierra en la vivienda indígena se asoció con un aumento de más de seis veces en la prevalencia del riesgo de desnutrición aguda $(6,25$ frente a $0,9 \%)$ y un aumento de más de veintiséis veces en la prevalencia de la desnutrición global $(11,0 \%$ vs $0,4 \%)$. Estos hallazgos quizás no sean tan sorprendentes, dado que estudios recientes han demostrado que los niños pequeños que viven en casas con piso de tierra tienen más probabilidades de estar infectados con parásitos intestinales que pueden causar diarrea y malnutrición ${ }^{(19)}$.

La presente investigación también mostró que los episodios de diarrea recientes, están relacionados con una prevalencia más alta del riesgo de desnutrición global y con una prevalencia más baja de sobrepeso. Y finalmente, teniendo en cuenta que la susceptibilidad a la infección también puede verse influenciada por la salud del niño al nacer, se usó la información sobre si el niño nació en un medio hospitalario y se encontró que un niño que nació en parto domiciliario, tuvo una prevalencia mayor de desnutrición crónica y de desnutrición global.

En cuanto a la educación, la población indígena quedó significativamente rezagada respecto del resto del país. El promedio de años de educación de la población mayor de 10 años fue solo de 2,2 años, en comparación con 7 años a nivel nacional. De manera similar, la tasa de analfabetismo de la población indígena alcanzó el $51 \%$ en comparación con el $7,1 \%$ a nivel nacional, siendo la tasa de analfabetismo más pronunciada en las áreas rurales $(53,3 \%)$ que en las urbanas $(29,2 \%)$. La familia de lengua Guaraní tuvo el mayor porcentaje de población "sin instrucción" (49,5\% en nuestra muestra, $41 \%$ en base a toda la encuesta).

Existe un fuerte vínculo entre la educación materna y la salud de los niños según varios reportes ${ }^{(15,16)}$. Los niños nacidos de mujeres educadas generalmente sufren menos de desnutrición. El presente estudio mostró que la prevalencia del riesgo de desnutrición crónica era un $60 \%$ mayor en los niños indígenas cuyas madres eran analfabetas.

La importancia de las prácticas de alimentación infantil para la nutrición infantil también está bien reconocida en la literatura sobre nutrición ${ }^{(12,13)}$. Se encontró que los niños que no fueron amamantados exclusivamente durante los primeros seis meses de vida mostraron una prevalencia de obesidad dos veces mayor que los niños que lo fueron. Además, se encontró que los niños amamantados (no necesariamente exclusivamente) durante los primeros doce meses de vida tenían una prevalencia siete veces menor de desnutrición aguda y una prevalencia de desnutrición global dos veces menor.

Por último, esta investigación también examinó la asociación entre el bajo peso al nacer y la desnutrición. El bajo peso al nacer está asociado con un rango de consecuencias adversas tanto a corto como a largo plazo, incluyendo un mayor riesgo de mortalidad infantil y de desnutrición. Se encontró una prevalencia de bajo peso del 15,3\% y una relación significativa entre el bajo peso al nacer y el riesgo de desnutrición global. Casi todos los niños con bajo peso $(81,7 \%)$ nacieron de madres no alfabetizadas (37,9\% de madres), lo que puede hacer el primer efecto similar de la madre sobre el riesgo de desnutrición global.

\section{CONCLUSIÓN}

Se constatan altas prevalencias de desnutrición crónica y de desnutrición global en niños indígenas hasta cuatro veces mayor que las prevalencias observadas en niños no indígenas. Las tasas de prevalencia de la desnutrición se asociaron con factores ambientales del hogar y factores socioeconómicos.

Existe una política de salud indígena que precisa fortalecerse e implementar estrategias de intervención preventivas, para evitar la desnutrición a edades tempranas y posibilitar el tratamiento oportuno para el desarrollo óptimo de todas las potencialidades del niño.

El perfil de salud nutricional de los niños indígenas en Paraguay amerita mejorar las estrategias de implementación de políticas e intervenciones de 
alimentación y nutrición diseñadas para los pueblos indígenas - por ejemplo, el Programa Alimentario Nutricional Integral (PANI), sustentado por la Ley 4698/12 “De Garantía Nutricional en la Primera Infancia", brinda a los niños indígenas menores de 5 años de edad un modelo universal de atención independientemente de su estado nutricional a través de entrega gratuita de leche enriquecida y de la prestación de todas las ofertas sanitarias (controles de crecimiento y desarrollo, inmunización, charlas educativas, entre otras). Estas políticas y programas deben seguir adaptándose de acuerdo con los estilos de vida culturales y las percepciones alimentarias de las comunidades indígenas para mejorar su situación actual.

\section{REFERENCIAS BIBLIOGRÁFICAS}

1. Lutter CK, Chaparro CM. Malnutrition in infants and young children in Latin America and the Caribbean: Achieving the Millennium Development Goals. Pan American Health Organization, Washington D.C. 2008.

2. Torres C. La equidad en materia de salud vista con enfoque étnico. Rev. Panam. Salud Pública. 2001;10:188-201.

3. Montenegro RA, Stephens C. Indigenous health in Latin America and the Caribbean. Lancet. 2006;367(9525):18591869. doi: 10.1016/S0140-6736(06)68808-9.

4. Horta B L, Santos R V, Welch J R, Cardoso A M, Vieira dos Santos J, Oliveira Assis A M, Lira P C I and Coimbra, Jr C E A. Nutritional status of indigenous children: Findings from the First National Survey of Indigenous People's Health and Nutrition in Brazil. Int J Equity Health. 2013 Apr 3;12:23. doi: 10.1186/1475-9276-12-23.

5.Martins VJB, Toledo Florêncio TMM, Grillo LP, et al. Long-Lasting Effects of Undernutrition. Int J Env Res Pub He. 2011 Jun;8(6):1817-1846. doi:10.3390/ijerph8061817.

6. San Sebastián M, Hurtig A-K. Review of health research on indigenous populations in Latin America, 1995-2004. Salud Pública Mex. 2007 Jul-Aug;49(4):316-320.

7. Mendoza L. El derecho al bienestar para la infancia indígena es una tarea pendiente de todos. Pediatr. (Asunción). 2015; 42(2):99-101.

\section{AGRADECIMIENTO}

Este estudio fue realizado en el marco del proyecto "La transición nutricional en el Paraguay: ¿En donde estamos?" (PINV 15-1304), el cual es ejecutado por el Instituto Desarrollo y financiado por el Consejo Nacional de Ciencias y Tecnología (CONACYT Paraguay), a través del programa PROCIENCIA, con recursos del Fondo para la Excelencia de laEducación e Investigación (FEEI) del Fondo Nacional de Inversión Pública y Desarrollo (FONACIDE).

También agradecemos al equipo técnico de la Dirección General de Estadística, Encuesta y Censos (DGEEC).

8. DGEEC. Principales Resultados EHI/2008, Encuesta de Hogares Indígenas. 2008. Dirección General de Estadística, Encuesta y Censos (DGEEC)

9. Rao JNK, Scott AJ. On chi-squared tests for multi way contingency tables with cell proportions estimated from survey data. Ann. Stat.1984 Mar ;12(1):46-60.

10. StataCorp. Stata survey data reference manual: Release 13. 2013. Stata Press Publication.

11. StataCorp. Stata: Release 13. Statistical Software. 2013. College Station, TX: Stata Corp LP.

12. World Health Organization. The World Health Organization's infant-feeding recommendations. Bull. WHO. 1995;73:165-174.

13. Brown K, Dewey K, Allen L. The World Health Organization's infant-feeding recommendations. Complementary Feeding of Young Children in Developing Countries: A Review of Current Scientific Knowledge. Switzerland: WHO Geneva; 1998.

14. Bern $\mathrm{C}$, et al. The magnitude of the global problem of diarrheal disease: A ten-year update. Bull. WHO. 1992;70:705-714.

15. Handa S. Maternal Education and Child Height. Econ Dev Cult Change. 1999 Jan;47(2):421-439. 
16. Frost MB, Forste R, Haas DW. Maternal education and child nutritional status in Bolivia: Finding the links. Soc. Sci.Med. 2005;60(2):395-407.20.

17. Sanabria MC. Análisis de la situación de salud infantil y antropometría en menores de 5 años. Asunción: DGEEC$\mathrm{EPH} ; 2005$.

18. Sanabria MC, Sánchez Bernal S, Osorio A, Bruno S.
Análisis de la situación nutricional de los niños menores de cinco años en Paraguay a partir de la encuesta de ingresos y gastos y de condiciones de vida 2011-2012. Asunción-Paraguay: UNICEF; 2014.

19. Cattaneo MD, Galiani S, Gertler PJ, Martinez S y Titiunik R. Housing, Health, and Happiness. Am. Econ. J: Econ Pol. 2009;1(1):75-105. 